\title{
The Influence of Repairs on the Stress Distribution and Compression Fracture Strength of Denture Bases
}

\author{
Morita Kimiyuki, DDS, PhD, Toyoda Minoru, DDS, PhD, \\ Takagaki Tatsuru, DDS, PhD and Ishida Masahiro, DDS, PhD \\ Department of Prosthetic Dentistry, Kanagawa Dental College \\ (Chief : Prof. Toyoda Minoru)
}

\section{Clinical significance}

This study examined the dynamic state of mandibular complete dentures after repair. It was found that stress concentrated in the midline area of the denture base. Dentures repaired with reinforcing wires did not regain their original strength. The results suggest that, once they are broken, repaired mandibular complete dentures tend to break again.

\section{ABSTRACT}

Purpose: Because of the shape of mandibular complete dentures, stress is the greatest along the midline. These dentures frequently fracture at or near this area. In order to contribute to the database of information available to dental professionals, the present study was undertaken to evaluate the distribution of stress and the fracture strength of denture bases, both before and after repair of a midline fracture.

Materials and Methods: Twelve denture bases (each $3 \mathrm{~mm}$ thick) were constructed of polymethyl methacrylate acrylic resin. The stress pattern of each base was characterized by measuring changes in thermal emission from the surface of the denture base. The fracture strength of six of the bases was measured by bending them with a compression-bending tester until they fractured.

Results : Stress concentration along the midline of the denture bases was observed. The mean fracture strengths of unbroken bases, repaired bases, and bases repaired with both resin and wire were 9.36 , 2.28 , and $4.34 \mathrm{~N}$, respectively.

Conclusion: Greater stress was observed along the midline in bases repaired with autopolymerizing resin only than in unbroken bases. In addition, the fracture strength of the repaired bases was only about $1 / 4$ that of unbroken bases. It was observed that stress was less concentrated along the midline of bases repaired with autopolymerizing resin and a reinforcement wire compared to either repaired bases or unbroken bases.

\section{Key words}

denture repair, thermoelastic stress analysis, stress distribution, reinforcing wire

Corresponding author: Morita Kimiyuki

82, Inaoka, Yokosuka 238-8580, Japan

Tel : +81-468-25-1500, Fax : +81-468-22-8861

E-mail : pdmorita $2000 @$ hotmail.com or pdmorita@ kdcnet.ac.jp

Received on January 18, 2002/Accepted on July 1, 2002 


\section{INTRODUCTION}

Many published studies ${ }^{1-7)}$ address the fracture of dental prostheses and their repair. However, there have been few reports on the properties of repaired dentures. Mandibular complete dentures frequently fracture along the midline $^{4)}$ for two reasons. First, when force is applied to the molar regions of these dentures, leverage multiplies the resultant force applied to the midline of the denture base. Second, stress depends upon cross-sectional area, and mandibular complete dentures must, for practical reasons, be thinner at the midline than in other areas of the denture. The cross-sectional area is further reduced along the midline because of the presence of notches ${ }^{3-6}$.

Denture bases repaired with autopolymerizing resin are reported to be less than half as strong as unbroken denture bases ${ }^{1,7)}$. There have been many studies on fracture of denture bases using morphologically simple ${ }^{7,8)}$ specimens. In order to clarify the mechanism of stress concentration by external forces and the effect of repairs on the distribution of stress, specimens that closely resemble actual mandibular dentures were tested in this study. A non-contact method $^{9-17)}$ was used to characterize the distribution of stress ${ }^{18,19)}$. The compression fracture strength of the repaired denture bases was measured and compared to that of unbroken denture bases.

\section{MATERIALS AND METHODS}

\section{Stress Analysis}

A plaster model of an edentulous mandible was fabricated by making an impression from a model (Model $402 \mathrm{~K}$, Nisshin, Kyoto, Japan) with vinyl polysiloxane impression material (Duplicone, Shofu, Kyoto, Japan). On each

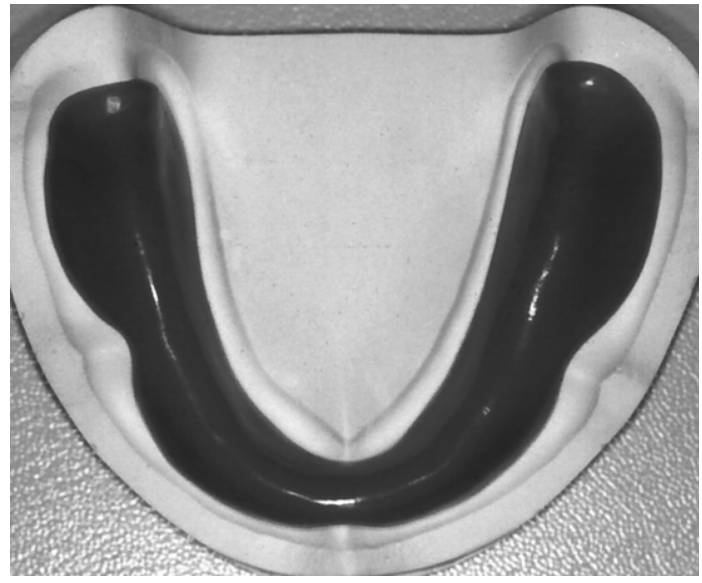

Fig. 1 Drawing of a denture base used for testing

stone cast, a vacuum-formed $3 \mathrm{~mm}$-thick base plate (Erkoplast-o, Erkodent, Pfalzgrafenweiler, Germany) was adapted to ensure uniformity of the denture base thickness.

Casts and base plates were invested with dental stone in metal denture flasks. The vacuum-formed base plates were discarded, and then heat-polymerizing acrylic resin $(0.43 \mathrm{~m} / / \mathrm{g})$ (Acron, GC Dental Products, Tokyo, Japan) was packed and processed. The mixture was polymerized by immersing it in cold water, gradually raising the water temperature to $100^{\circ} \mathrm{C}$ over a 30 min period, maintaining the boil for another 30 minutes, removing the heat source, and finally letting the polymerized resin stand for 12 hours (GC Auto Cure System, GC Dental Products). The completed resin bases were used in these experiments (hereinafter referred to as PMMA). All the PMMAs were prepared at $23 \pm 2^{\circ} \mathrm{C}(50 \pm$ $10 \%$ relative humidity) and tested in accordance with ISO 156720) (Fig. 1).

To determine the experimental conditions making it the easiest to confirm changes in stress distribution, different parts of the PMMAs were held in a vise, and vibrations were applied from a variety of directions. The most satisfactory results were obtained when 1) The posterior margin of the right side of the denture base was 


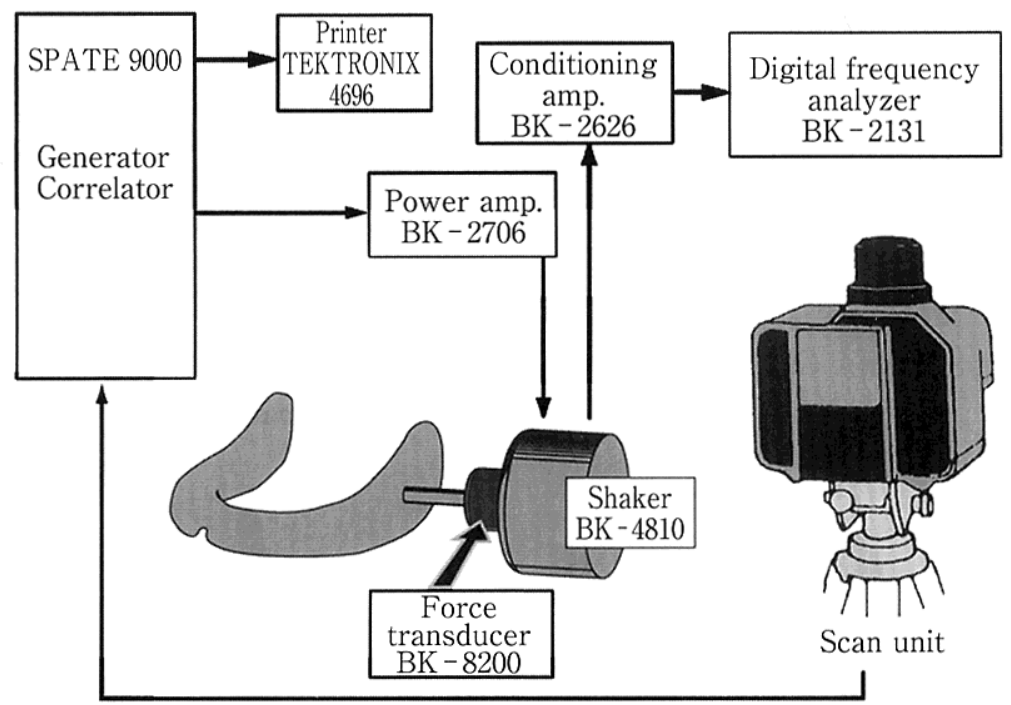

Fig. 2 Block diagram of the stress analysis system (SPATE)

held in the vise, 2) Vibrations were applied to the first molar region on the left side from the buccal aspect, and 3) Stress was measured from the lingual aspect. Changes in stress distribution were most easily measured when vibrations were applied at $0.3 \mathrm{G}$ and $30 \mathrm{~Hz}$.

To establish a reference, images of the stress distribution on the surface of six PMMAs were produced with a scanning infrared radiometer (SPATE-9000, Ometron, London, UK). The SPATE (Stress pattern analysis by thermal emission) system detects the infrared flux emitted from a surface as a result of minute temperature changes (as small as $0.001 \mathrm{~K}$ ) in a cyclically stressed structure ${ }^{9-16)}$. A block diagram of the instrument is shown in Fig. 2. This measuring instrument is based on the principle of thermoelasticity as confirmed by Lord Kelvin ${ }^{17)}$ in 1835. Thermoelastic stress analysis is a method used in various fields to evaluate stress. SPATE is a system particularly known for its reliability and is being used in NASA ${ }^{16)}$ and in medical institutions ${ }^{13,15)}$. In dentistry, it is used for measuring pressure exerted on dentures ${ }^{15}$. To insure uniform emissivity of specimen surfaces, they were painted flat black (Asahipen, Osaka, Japan).
After measurements were completed, these six bases were cut in half, and a $2 \mathrm{~mm}$ strip was removed from each half along the midline. The bases were repaired with a gypsum core and autopolymerizing acrylic resin (Unifast II, GC Dental Products) according to the method described by Bailey ${ }^{211}$. Images of the stress distribution of the repaired denture bases (REPs) were again produced.

Next, grooves were cut at intervals of $3-4$ $\mathrm{mm}$ in the REP lingually from the right second premolar to the left second premolar. Wax $(0.4$ $\mathrm{mm}$ thick) was applied to the plaster model. $\mathrm{Co}-\mathrm{Cr}$ reinforcing wires (1.2 $\mathrm{mm}$ diameter) (Sankin, Tokyo, Japan) were placed and then the wires were air-particle abraded with $50 \mu \mathrm{m}$ aluminum oxide (particles $4 \mathrm{kgf} / \mathrm{cm}^{2}, 10 \mathrm{sec}$ ). Preparations were made for embedding the wires evenly in the grooves. A core was made with silicone putty (Exafin, GC Dental Products). With the core as a guide, the grooves of the remaining specimens were adjusted. The wirereinforced base was repaired with autopolymerizing resin (Unifast II). Hereinafter, the repaired base will be referred to as "BAR." After all these specimens were completed, their thickness was adjusted, and the stress distribution was 


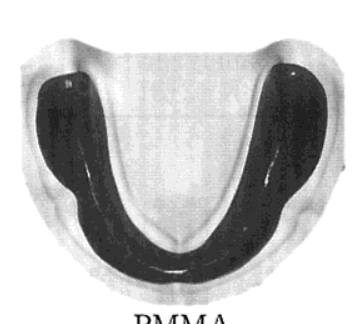

PMMA

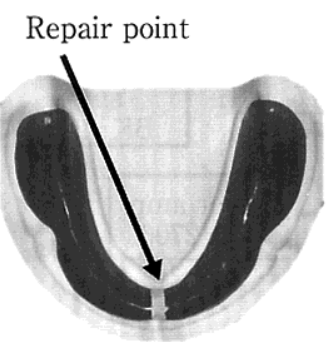

REP

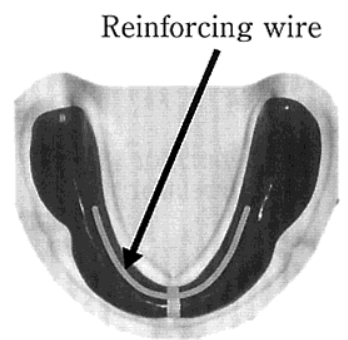

BAR

Fig. 3 Schematic illustration of test materials

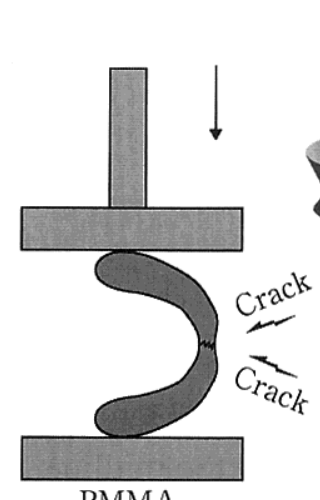

PMMA

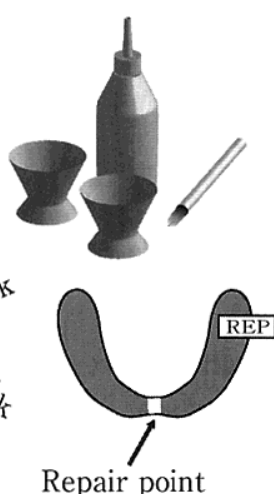

Repair point

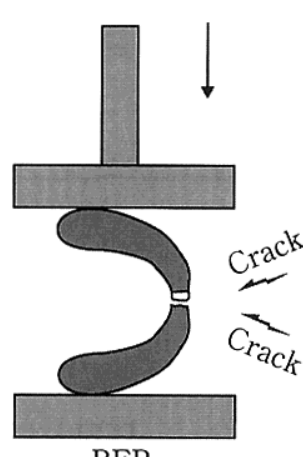

REP

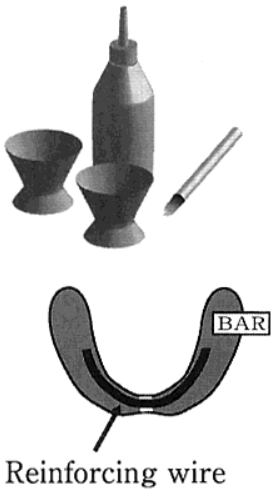

Reinforcing wire

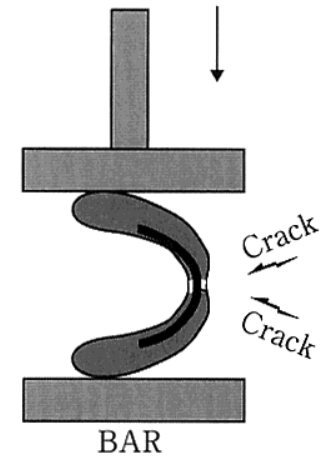

BAR

Fig. 4 Illustration of the compression fracture test

measured (Fig. 3). Stress distributions were then characterized with the scanning infrared radiometer (SPATE-9000).

\section{Compression Fracture Test}

The fracture strength of denture bases, including PMMAs, REPs, and BARs, was measured with a compression bending tester (AGS-1000 B, Shimadzu, Kyoto, Japan). The right-side margin of each denture base was clamped onto the table of the tester with a vise, and load was applied to the left-side margin from the buccal aspect (Fig. 4). The test was run at a speed of $1 \mathrm{~mm} / \mathrm{min}$. The data thus obtained were analyzed by one-way analysis of variance, and the mean values were compared by a post hoc Fisher's protected-LSD test, for which significance levels were set at $\mathrm{p}<0.01$.

\section{RESULTS}

The stress patterns (Fig. 5) observed in the six PMMAs tested were qualitatively identical. Stress was concentrated in the midline area from the left canine to the right canine, confirming prior reports ${ }^{18,19)}$ of midline stress in mandibular complete dentures. Stress observed in REPS was also concentrated along the midline, and it was concentrated into an even smaller region than it was in the PMMAs. The stress pattern observed in BARs showed that stress was spread over a much larger portion of the denture base. Stress was noticeably less concentrated along the midline.

The results of the compression fracture tests were $9.36 \pm 1.75 \mathrm{~N}$ for PMMAs; $2.28 \pm 0.31 \mathrm{~N}$ for REPs ; and $4.34 \pm 0.69 \mathrm{~N}$ for BARs. Significant differences $(p<0.01)$ were found among all the specimens (Fig. 6). 


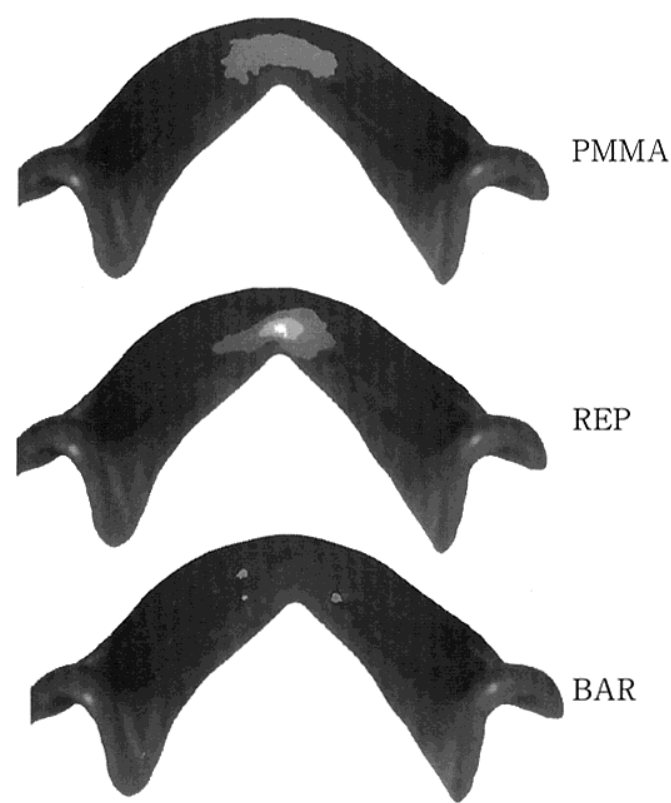

Fig. 5 Results of stress analysis In PMMAs, stress was concentrated along the midline. Greater stress was concentrated along the midline in REPs than in PMMAs. Stress was less concentrated along the midline in the BARs

\section{DISCUSSION}

Mandibular complete dentures are the weakest where there are notches and pinholes, and at junctions between dissimilar materials ${ }^{3-6,22)}$. However, these dentures break most often because stress concentrates in a weak region of the denture base. Such concentrations are the greatest where the cross-sectional area is at a minimum. Leverage concentrates stress into the region near the midline of mandibular complete dentures; consequently, most repairs of these dentures must be made along the midline $e^{4)}$. Dentures that contain lingual or labial notches in the midline, cut to accommodate frenula, are especially vulnerable to fracturing or cracking ${ }^{6}$. To lessen the risk of fracture, notches should be made as small as possible, and they should be rounded rather than sharp-angled.

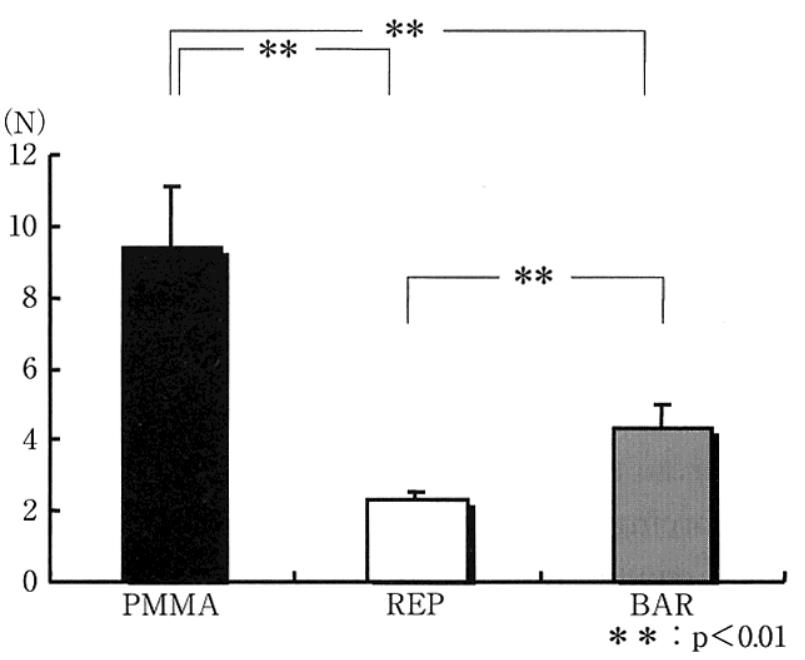

Fig. 6 Results of compression fracture tests

The stress analysis presented here shows that stress is concentrated along the midline of the specimens, and the fracture tests showed that all the specimens fractured along the midline connecting lingual to labial notches. These findings indicate that the midline area is the most fragile part of the mandibular complete denture.

Previously, we performed a modal analysis using the same specimens ${ }^{18)}$ and mandibular complete dentures ${ }^{19)}$ (under vertical loading). We reported there is a strong likelihood that stress is concentrated on the median area of the mandibular complete denture because of distortion of the denture base at the midline ${ }^{18,19}$.

In BARs, it was observed that stress was spread throughout the denture base. At the same time, there was a smaller concentration of stress along the midline. This finding is most likely attributable to the reinforcing wires that distribute stress along their full length. Although BARs were significantly stronger than REPs $(p<0.01)$, they were not as strong as unbroken denture bases.

In our experiment, all BAR specimens fractured on the labial side where no reinforcing wires were placed. In order for a repaired denture base to possess the same fracture strength as an unbroken denture base, the repair 
process would have to include making the denture base thicker in the midline region, which would be unacceptable for most patients. Gainsworth ${ }^{23)}$ reported cases in which reinforcements placed in the resin base failed to fulfill their purpose, as breakage occurred at the very place where wires were embedded. Darbar ${ }^{4)}$ et al. reported that fracture lines detoured around reinforcing wires. The results of the present study suggest that reinforcing wires are helpful if the resin base is thick enough to disperse stress.

The standard deviation of the mean fracture strength of PMMAs $(1.75 \mathrm{~N})$ was relatively large because of large individual differences among the specimens, which exhibited a variety of shapes, just as actual dentures are idiosyncratic. In a previous study ${ }^{1)}$ of maxillary dentures, the standard deviations were about 16 to $21 \%$, and the strength of the specimens repaired only with autopolymerizing acrylic resin and with reinforcements was about 18 and $66 \%$ of that of the original specimens, respectively. The results were similar to those obtained in the present study.

The measuring instruments used in this study did not allow fatigue of test materials to be included in the experimental design. In the future, further experiments taking fatigue into account will be done in this laboratory, as well as experiments with various other types of repair materials that are available.

\section{SUMMARY}

After mandibular complete dentures were broken along the midline, they were repaired with autopolymerizing resin using the conventional method. The effect of repairs on the distribution of stress in the resin base and the fracture strength of each denture base were examined. It was found that:

1. Stress is concentrated along the midline of the denture base by external forces.

2. In broken dentures repaired only with autopolymerizing acrylic resin, there is more stress along the midline than there is in unbroken dentures.

3. Repaired dentures are weaker than unbroken dentures.

4. When midline repairs include the placement of a reinforcing wire, stress is dispersed. However, these repaired dentures were only about half as strong as unbroken denture bases. When the repaired denture bases were fracturetested, all fractured on the labial aspect where there were no reinforcing wires.

\section{REFERENCES}

1) Polyzois GL, Andreopoulos AG, Lagouvardos PE Acrylic resin repair with adhesive resin and metal wires : Effects on strength parameters. J Prosthet Dent $75:$ 381-387, 1996.

2) Beyli MS, von Fraunhofer JA. Repair of fracture of acrylic resin. J Prosthet Dent $44:$ 497-503, 1980.

3) Kelly F. Fatigue failure in denture base polymers. J Prosth Dent $21: 257-266,1969$.

4) Darbar UR, Huggett R, Harrison A. Denture fracture-a survey. Br Dent J $176: 342-345,1994$.

5) Glantz P-O, Stafford GD. Clinical deformation of maxillary complete dentures. J Dent $11: 224-230$, 1983.

6) Beyli MS, von Fraunhofer JA. An analysis of causes of fracture of acrylic resin dentures. J Prosthet Dent $46: 238-241,1981$.

7) Ward JE, Moon PC, Levine RA et al. Effect of repair surface design, repair material, and processing method on the transverse strength of repaired acrylic denture resin. J Prosthet Dent 67 : 815-820, 1992.

8) Stipho HD, Stipho AS. Effectiveness and durability of repaired acrylic resin joints. J Prosthet Dent 58 : 249-253, 1987.

9) Dulieu-Barton JM, Stanley P. Development and applications of thermoelastic stress analysis. J Strain Analysis 33 : 93-104, 1998.

10) Dulieu-Barton JM. Introduction to thermoelastic stress analysis. Strain $35: 35-39,1999$.

11) Stanley P, Chan WK. Quantitative stress analysis by means of the thermoelastic effect. J Strain Analysis 
$20:$ 129-137, 1985.

12) Baker LK, Webber MB. Thermoelastic stress analysis. Optica Acta 29 : 555-563, 1982.

13) Vanderby R Jr, Kohles SS. Thermographic stress analysis in cortical bone. J Biomechanical Engineering 113 : 418-422, 1991.

14) Dulieu-Barton JM, Stanley P. Application of thermoelastic stress analysis to composite materials. Strain 35:41-48, 1999.

15) Toyoda M, Kasori T, Hama A et al. Change of stress bearing areas in abutment teeth and denture-foundation areas caused by application of impact to different points. Bull Kanagawa Dent Coll 23:87-92, 1995.

16) Gyekenyesi AL, Baaklini GY. Thermoelastic stress analysis: The mean stress effect in metallic alloys. NASA/TM-1999-209376, 1-11, Washington DC : NASA Center for AeroSpace Information, 1999.

17) Thomson W (Lord Kelvin). On the thermoelastic, thermomagnetic and pyro-electric properties of matter. Phil Mag 5:4-27, 1878.

18) Toyoda M, Morita K, Imai M et al. A study on the influence of denture base repair upon the dynamic characteristics of denture base. J Jpn Prosthodont Soc $44: 65-70,2000$. (in Japanese)

19) Mizutani $T$, Morita K. Influence of the different on denture base materials toward the movement of dentures. J Kanagawa Odontological Soc 28: 336-355, 1993. (in Japanese)

20) ISO 1567, 3 rd edition. 1-19, Geneve : International Organization for Standardization, 1999.

21) Bailey LR. Denture repairs. Dent Clin North Am 19 : 357-366, 1975.

22) Davies BJ. Handbook of mechanical engineering 116-121, London : Springer-Verlag, 1980.

23) Gainsworth BL. Space control. J Dent Child 4 th Quarter : 188-194, 1955. 